\title{
EVALUATION OF LOCAL ORIENTATION FOR TEXTURE CLASSIFICATION
}

\author{
Dana Elena Ilea, Ovidiu Ghita, Paul F. Whelan \\ Vision Systems Group, Dublin City University, Glasnevin, Dublin 9, Ireland \\ danailea@eeng.dcu.ie,ghitao@eeng.dcu.ie,whelanp@eeng.dcu.ie
}

Keywords: Texture, image orientation, local distributions, observation scale, classification.

\begin{abstract}
The aim of this paper is to present a study where we evaluate the optimal inclusion of the texture orientation in the classification process. In this paper the orientation for each pixel in the image is extracted using the partial derivatives of the Gaussian function and the main focus of our work is centred on the evaluation of the local dominant orientation (which is calculated by combining the magnitude and local orientation) on the classification results. While the dominant orientation of the texture depends strongly on the observation scale, in this paper we propose to evaluate the macro-texture by calculating the distribution of the dominant orientations for all pixels in the image that sample the texture at micro-level. The experimental results were conducted on standard texture databases and the results indicate that the dominant orientation calculated at micro-level is an appropriate measure for texture description.
\end{abstract}

\section{INTRODUCTION}

Texture is a fundamental property of digital images that has received a significant amount of attention from the computer vision community. This is motivated by the fact that the objects in digital images are not characterised only by their shapes but also by the structural relationship between the pixels that define their imaged surfaces. Based on this observation, a large number of studies have been dedicated to the development of a robust texture descriptor that is able to adapt to the local characteristics in the image. In this sense, the texture has been analysed based on the model that has been employed to describe it. While there is not a widely accepted definition for texture in the vision community, most of the developed techniques approach the texture either from a structural or a statistical perspective (Haralick, 1979; Dyer et al, 1980). In statistical approaches, the texture is described by the spatial distribution of the pixels in the image (Chellappa et al, 1998) while in structural approaches the texture is defined as a relational arrangement of texture primitives (Materka and Strzelecki, 1998; Petrou and Sevilla, 2006). In this paper we propose a hybrid statistical-structural approach where the texture is described in terms of the distribution of orientations calculated at microlevel for all pixels in the image. The distributions of edge orientations have been previously used for image segmentation (Flores and Leon, 2003; Ojala et al, 2002-a; Liu and Wang, 2003), retrieval (Zhou et al, 2003; Manjunath and Ma, 1996) and tracking (Marimon and Touradj, 2007) while our aim is to evaluate the robustness of these measures when applied to texture classification. While the orientation of the texture is highly influenced by the observation scale, in this work we attempt to quantify the importance of the scale and size of the texture unit (where the local dominant orientation is calculated) on the classification results. This paper is organised as follows. Section 2 describes the estimation of the dominant orientation in the image. Section 3 details the implementation of the texture classification framework. Section 4 describes the experimental results while in Section 5 are provided concluding remarks.

\section{ESTIMATION OF EDGE ORIENTATION}

The local orientation in the image is obtained by calculating the first derivatives in two orthogonal 
directions (Kass and Witkin, 1987) and the edge orientation can be simply determined using the expression illustrated in equation 1 .

$$
\nabla f=\left[\frac{\partial F}{\partial x}, \frac{\partial F}{\partial y}\right], \quad \Theta=\operatorname{arctg}\left(\frac{\partial F / \partial y}{\partial F / \partial x}\right)
$$

where $\nabla$ defines the gradient operator and $\Theta$ is the edge orientation. The gradient is computed for each pixel in the image and this operation is traditionally carried out by filtering the image with operators such as Sobel in the $x$ and $y$ directions. The estimation of the gradient using small kernels is not appropriate since this operation is sensitive to image noise. Thus, in this paper we have calculated the partial derivatives in the image by filtering the image in the $x$ and $y$ directions with the filters that implement the derivatives of the Gaussian function. This is achieved by filtering the image with 1dimensional (1D) operators that are calculated using the expression illustrated in equation 2 .

$$
G(x)=\frac{1}{\sqrt{2 \pi} \sigma} e^{-\frac{x^{2}}{2 \sigma^{2}}}, \quad \frac{\partial G}{\partial x}=-\frac{x}{\sigma^{2}} G(x)
$$

where $\sigma$ is the scale of the Gaussian function (see Figure 1 for a plot of the derivative of Gaussian function for different scale parameters. For clarity purposes the plots illustrated in Figure 1 were generated by varying the parameter $x$ in equation 2 in the interval $[-50,50])$.

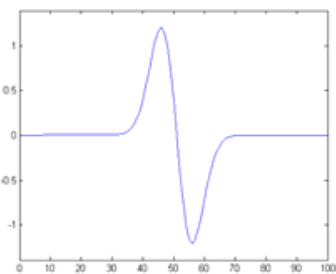

(a)

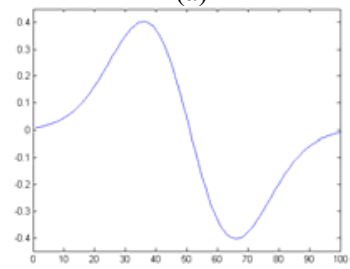

(c) (b)

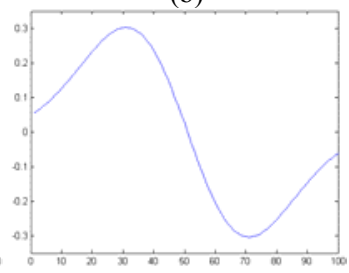

(d)

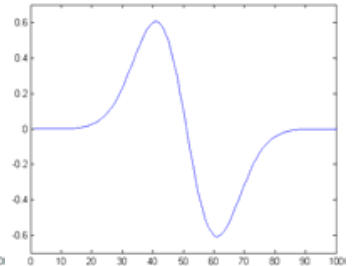

Figure 1: The derivative of Gaussian function. (a) $\sigma=0.5$. (b) $\sigma=1.0$. (c) $\sigma=1.5$. (d) $\sigma=2.0$.
The main advantage of the calculation of the partial derivatives using the derivative of the Gaussian resides in the fact that the Gaussian has a smoothing effect and the scale parameter controls the amount of noise reduction. After the calculation of the partial derivatives, the weak edge responses were eliminated by applying a non-maxima suppression procedure (similar to that described in (Canny, 1986)) and the edge orientation is determined for each pixel using the expression illustrated in equation 1 . The distributions of the edge orientations calculated for two different textures from the Outex texture database (Ojala et al, 2002-b) are illustrated in Figure 2.
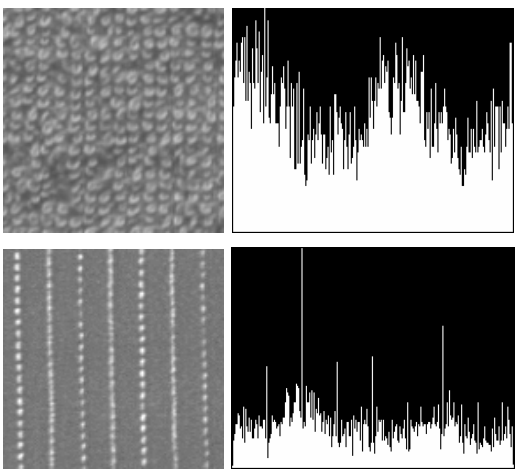

Figure 2: Distributions of edge orientation calculated for two textures (top - isotropic and bottom - oriented) from the Outex database (Ojala et al, 2002-b).

The distribution of the edge orientations illustrated in Figure 2 provides the primary discrimination between textures. Its main disadvantage is the fact that this distribution is calculated over the entire image and is not able to robustly sample the texture orientation at micro-level. Thus, in this paper we propose to evaluate the dominant orientation of the texture calculated at micro-level for all texture units that are defined as the local neighbourhood around each pixel in the image. The distribution of the dominant orientations calculated for all texture units is then employed to capture the local orientation of the texture at macro-level.

\subsection{Estimation of the Dominant Orientation of Texture at Micro and Macro-Level}

The problem of analysing the texture orientation at a given observation scale is not a straightforward task as the orientation of the texture may be isotropic at 
macro level but having strong orientation at microlevel. This problem has been addressed in the paper by Germain et al, 2003 where they analysed a texture that is formed by sinusoidal curves having the same period. In this regard, when the texture is evaluated at a micro-level in a small neighbourhood (say $3 \times 3$ or $5 \times 5$ ) the texture appears to have a strong orientation. But when it is analysed at macro-level the texture appears to be isotropic. This process can be clearly visualised in Figure 3.

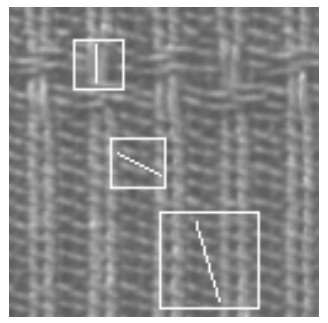

Figure 3: The variation of the orientation of texture at different observation scales.

The next problem is how to extract the dominant orientation of the texture in a local $k \times k$ neighbourhood. In our implementation we elected to determine the orientation of the texture by constructing the histogram of orientations for all pixels in the local neighbourhood and the dominant orientation is selected as the dominant peak in the histogram as follows,

$$
\begin{gathered}
H_{\Theta}=\bigcup_{i \in D} h_{\Theta}(i), h_{\Theta}(i)=\sum_{y=-k / 2}^{k / 2} \sum_{x=-k / 2}^{k / 2} \delta(\Theta(x, y), i), \\
\text { where } D \in[0,2 \pi], \delta(i, j)= \begin{cases}1 & i=j \\
0 & i \neq j\end{cases} \\
\Theta_{d}=\arg \max \left(H_{\Theta}\right)
\end{gathered}
$$

where $\Theta$ is the local orientation, $H_{\Theta}$ is the distribution of local orientations and $\Theta_{d}$ is the dominant orientation of the texture in the neighbourhood $k \times k$

\section{TEXTURE CLASSIFICATION}

The dominant orientation in the image is a powerful local texture descriptor but it is not robust in sampling the difference between textures that are subjected to illumination variation and image inversion. Thus, we need to augment the local texture orientation with measures such as local orientation coherence and contrast that are calculated in the local neighbourhood $k \times k$ where the dominant orientation of the texture has been estimated. In our implementation, the contrast measure is sampled by the mean gray-scale value calculated in the $k \times k$ neighbourhood and the orientation coherence $\left(\Theta_{c}\right)$ is calculated using the weighted standard deviation of the edge orientation of all pixels in the neighbourhood as follows:

$$
\Theta_{c}(i, j)=\sum_{m, n \in w_{k \times k}} \sqrt{\frac{\left(g_{x}^{2}+g_{y}^{2}\right)}{k^{2}}\left(\Theta_{m, n \in w_{k \times k}}-\Theta_{a v e}\right)^{2}}
$$

where $(i, j)$ are the coordinates of the pixel of interest, $g_{x}=\frac{\partial F}{\partial x}, g_{y}=\frac{\partial F}{\partial y}$ are the partial derivatives calculated for all pixels in the window $w_{k \times k}$ and $\Theta_{a v e}$ is the average edge orientation calculated for all pixels in the window $w_{k \times k}$. From the $k \times k$ neighbourhood around each pixel in the image three measures are calculated, namely the dominant orientation $\left(\Theta_{d}\right)$, the contrast value (C) and the orientation coherence $\left(\Theta_{c}\right)$. It is useful to mention that these three measures define the texture composition at micro-level while their distributions calculated over the entire image define the features that describe the texture at macro-level. This process is illustrated in Figure 4.

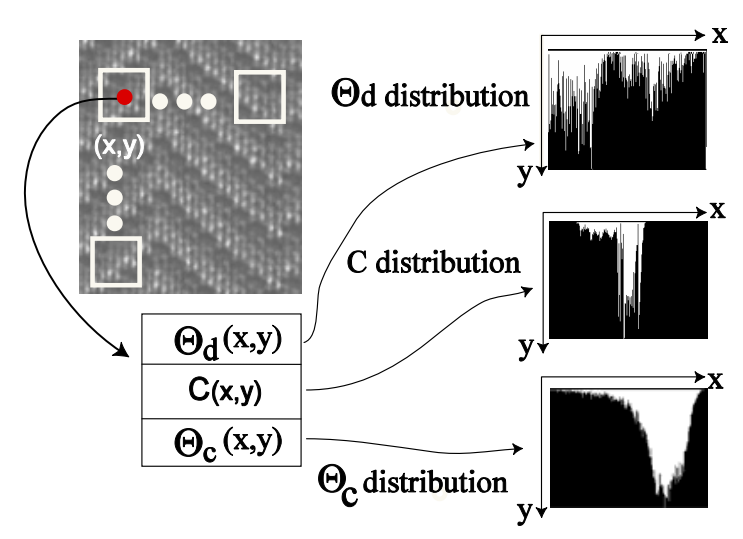

Figure 4: The calculation of the dominant orientation, contrast and orientation coherence distributions. 
To sample the image at different observation scales, the orientation, contrast and orientation coherence distributions are calculated for different values of the window parameter $k$. In our implementation the size of the local window is varied from $3 \times 3$ to $11 \times 11$. These distributions are concatenated into a feature vector that is used to train an SVM classification scheme (Chang and Lin, 2001). Figure 5 illustrates the shape of the macro-level distributions when the dominant orientation is calculated for all pixels in the image in neighbourhoods of differing sizes. It can be noticed that the distribution of the local dominant orientations in the image shows apparent peaks when calculated in small neighbourhoods $(3 \times 3)$ and shows an even distribution of the dominant orientations when calculated in larger neighbourhoods such as $7 \times 7$ and $11 \times 11$. This is motivated by the fact that the dominant orientation calculated in small neighbourhoods samples the local orientation of the texture while the dominant orientation when calculated in larger neighbourhoods tends to sample the anisotropic character of the texture with the increase in the size of the neighbourhood.

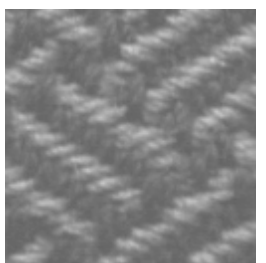

(a)

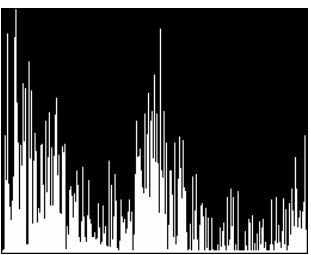

(c)

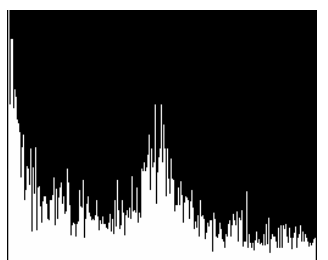

(b)

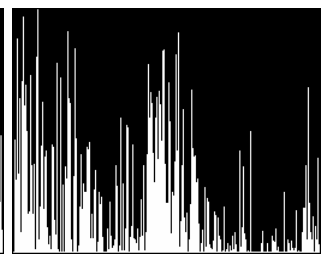

(d)
Figure 5: The distribution of the dominant orientations when the window parameter $k$ is varied. (a) Input texture image from Outex database. Distribution of the dominant orientation for texture units in (b) $3 \times 3$, (c) $7 \times 7$ and (d) $11 \times 11$ neighbourhoods.

\section{EXPERIMENTS AND RESULTS}

The experimental results in this paper were conducted on three Outex databases (TC 00000, TC
00001 and TC 00002) and on databases that were constructed by splitting recursively the images contained in the Brodatz database (Brodatz, 1966). The Outex databases are formed by 24 classes of standard textures as illustrated in Figure 6 (canvas, carpet and tile).
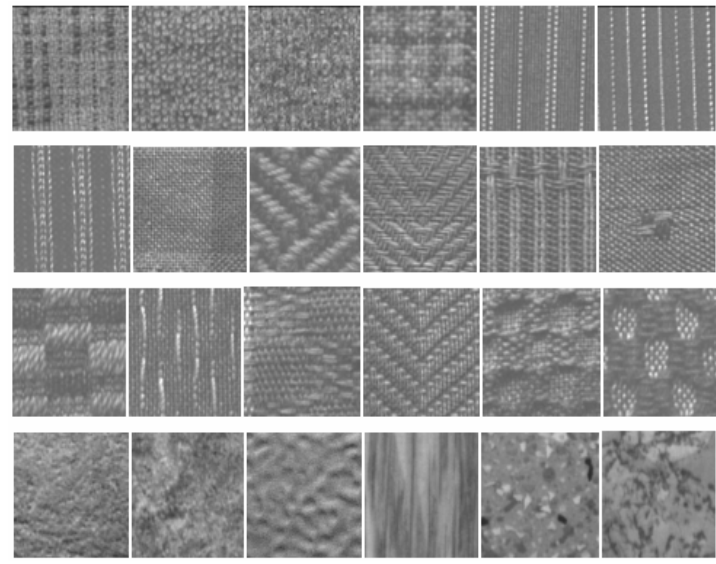

Figure 6: The 24 textures contained in the Outex database (Ojala et al, 2002-b).

The database TC 00000 consists of 480 texture images where the image size is $128 \times 128$. Database TC 00001 is formed by splitting the images that form the database TC 00000 in four parts and this results in 2212 texture images where the image size is $64 \times 64$. Database TC 00002 is obtained by splitting the texture images contained in the database TC 00001 into four parts and this generates 8832 images where the image size is $32 \times 32$.
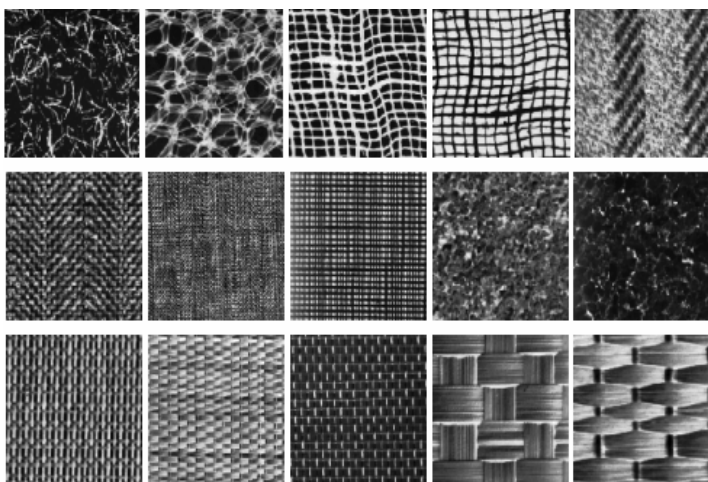

Figure 7: Samples of the Brodatz (Brodatz, 1966) textures used in our experiments. 
The Brodatz database (Brodatz, 1966) used in our study consists in a set of 36 texture images. This database is formed by near-isotropic textures and the original images were split in 4 (database BD 00000), 16 (database BD 00001) and 64 sub-images (database BD 00002). Database BD 00000 consists of 144 texture images (image size: $256 \times 256$ ), database BD 00001 has 576 texture images (image size: $128 \times 128$ ) and database $\mathrm{BD} 00002$ comprises 2304 images (image size: $64 \times 64$ ). A number of Brodatz textures used in our experiments are depicted in Figure 7.

In our experiments half of the images contained in each database were used for training while the remaining half was used for testing. As an example, when the experiments were conducted using the Outex TC 00000 database, 240 images were used for training and 240 images were used for testing. As indicated earlier, the similarity between the training and test textures was evaluated using an SVM classification scheme where polynomial kernels were employed to map the feature space. In this study we have conducted a number of experiments to evaluate the discriminative power of the texture features described in the previous section when the size of the texture images and the size and the number of windows applied to sample the texture orientation are varied. Also a number of tests were conducted to determine the optimal value of the $\sigma$ parameter that controls the scale of the derivative of Gaussian function that is applied to compute the partial derivatives in the image.

\subsection{Influence of the Parameter $\sigma$ on the Classification Results}

The aim of these tests is to evaluate the influence of the parameter $\sigma$ (that sets the scale of the derivative of the Gaussian function) on the classification results. To isolate the effect of this parameter on the classification results, the feature vector that describes the texture is formed only by the distribution of the dominant orientations when calculated in the smallest $(3 \times 3)$ neighbourhood. The classification results when applied to the Outex and Brodatz databases are illustrated in Table 1 (to limit the size of Table 1, the results of the experiments are reported only for databases TC 00000 , TC 00001 , BD 00000 and BD 00001).
Table 1: The influence of the parameter $\sigma$ (scale of the derivative of Gaussian function) on the classification results.

\begin{tabular}{|c|c|c|}
\hline Database & Parameter $\boldsymbol{\sigma}$ & Accuracy [\%] \\
\hline \multirow{3}{*}{$\begin{array}{c}\text { TC 00000 } \\
(128 \times 128)\end{array}$} & 0.5 & 91.66 \\
\cline { 2 - 3 } & 1.0 & 95.00 \\
\cline { 2 - 3 } & 1.5 & 92.91 \\
\cline { 2 - 3 } & 2.0 & 89.58 \\
\hline \multirow{4}{*}{$\begin{array}{c}\text { TC 00001 } \\
(64 \times 64)\end{array}$} & 2.5 & 80.83 \\
\cline { 2 - 3 } & 0.5 & 78.21 \\
\cline { 2 - 3 } & 1.0 & 69.78 \\
\cline { 2 - 3 } & 1.5 & 65.43 \\
\hline \multirow{3}{*}{$\begin{array}{c}\text { BD 00000 } \\
(256 \times 256)\end{array}$} & 2.0 & 47.91 \\
\cline { 2 - 3 } & 2.5 & 33.33 \\
\cline { 2 - 3 } & 0.5 & 91.66 \\
\cline { 2 - 3 } & 1.0 & 83.33 \\
\hline \multirow{4}{*}{$\begin{array}{c}\text { BD 00001 } \\
(128 \times 128)\end{array}$} & 1.5 & 75.00 \\
\cline { 2 - 3 } & 2.0 & 72.22 \\
\cline { 2 - 3 } & 2.5 & 81.94 \\
\cline { 2 - 3 } & 0.5 & 94.44 \\
\cline { 2 - 3 } & 1.0 & 93.40 \\
\hline
\end{tabular}

These experimental results (see Table 1) indicate that the best classification results are obtained when the scale parameter $\sigma$ is set to low values in the range $[0.5,1]$. There are two reasons behind these results. The first is motivated by the fact that with the increase value of the scale parameter the edges derived from weak textures are eliminated and the second reason consists in the requirement to increase the size of the derivative of Gaussian filters with the increase of the scale parameter $\sigma$ (this can be clearly observed in Table 1 where the classification accuracy is drastically reduced when the dominant orientation is calculated for images with a small size such as those contained in database TC 00001).

Based on the results depicted in Table 1, we have decided to set the scale parameter $\sigma$ of the derivative of Gaussian operator to 0.5 to limit the impact of undesired texture filtering and the windowing effects caused by the convolution with large kernels. 


\subsection{Classification Results when the Window Size is Varied}

A second set of experiments was conducted to evaluate the influence of the window size $w_{k \times k}$ on the classification results. To isolate the effect of the window size, we have conducted the experiments where the dominant orientation distribution was used alone (Table 2) and to evaluate the complementary character of the texture distributions discussed in Section 3, the dominant orientation distribution was used in conjunction with the contrast and orientation coherence distributions (Table 3).

Table 2: The influence of the widow size on the classification results (only the dominant orientation distribution is employed in the classification process).

\begin{tabular}{|c|c|c|}
\hline Database & Window size & Accuracy [\%] \\
\hline \multirow{2}{*}{$\begin{array}{c}\text { TC 00000 } \\
(128 \times 128)\end{array}$} & $3 \times 3$ & 91.66 \\
\cline { 2 - 3 } & $7 \times 7$ & 82.91 \\
\hline \multirow{2}{*}{$\begin{array}{c}\text { TC 00001 } \\
(64 \times 64)\end{array}$} & $11 \times 11$ & 71.25 \\
\cline { 2 - 3 } & $3 \times 3$ & 78.21 \\
\cline { 2 - 3 } & $11 \times 11$ & 65.71 \\
\hline \multirow{2}{*}{$\begin{array}{c}\text { TC } 00002 \\
(32 \times 32)\end{array}$} & $3 \times 3$ & 55.58 \\
\cline { 2 - 3 } & $7 \times 7$ & 45.48 \\
\hline \multirow{2}{*}{$\begin{array}{c}\text { BD 00000 } \\
(256 \times 256)\end{array}$} & $11 \times 11$ & 33.69 \\
\cline { 2 - 3 } & $3 \times 3$ & 28.39 \\
\hline \multirow{2}{*}{$\begin{array}{c}\text { BD } 00001 \\
(128 \times 128)\end{array}$} & $11 \times 11$ & 91.66 \\
\cline { 2 - 3 } & $3 \times 3$ & 66.66 \\
\cline { 2 - 3 } & $11 \times 7$ & 63.88 \\
\hline BD 00002 & $3 \times 3$ & 84.44 \\
\cline { 2 - 3 }$(64 \times 64)$ & $7 \times 7$ & 82.63 \\
\cline { 2 - 3 } & $11 \times 11$ & 67.73 \\
\hline
\end{tabular}

The experimental results depicted in Table 2 indicate that the discriminative power of the local dominant orientation distribution decreases with the increase of the window size. These results were expected since the local orientations calculated for larger windows sample the anisotropic properties of the texture. Another important conclusion resulting from the tests depicted in Table 2 is that the dominant orientation distribution is not efficient in sampling the properties of small textures (see the classification results obtained for database TC 00002).
Table 3: The influence of the widow size on the classification results (dominant orientation, contrast and orientation coherence distributions are employed in the classification process - see Section 3).

\begin{tabular}{|c|c|c|}
\hline Database & Window size & Accuracy [\%] \\
\hline \multirow{2}{*}{$\begin{array}{c}\text { TC } 00000 \\
(128 \times 128)\end{array}$} & $3 \times 3$ & 97.08 \\
\cline { 2 - 3 } & $7 \times 7$ & 95.00 \\
\hline \multirow{2}{*}{$\begin{array}{c}\text { TC 00001 } \\
(64 \times 64)\end{array}$} & $11 \times 11$ & 84.16 \\
\cline { 2 - 3 } & $3 \times 3$ & 95.54 \\
\hline \multirow{2}{*}{$\begin{array}{c}\text { TC 00002 } \\
(32 \times 32)\end{array}$} & $11 \times 11$ & 87.50 \\
\cline { 2 - 3 } & $3 \times 3$ & 78.21 \\
\hline \multirow{2}{*}{$\begin{array}{c}\text { BD 00000 } \\
(256 \times 256)\end{array}$} & $11 \times 11$ & 70.01 \\
\cline { 2 - 3 } & $3 \times 3$ & 60.19 \\
\cline { 2 - 3 } & $11 \times 7$ & 45.40 \\
\hline \multirow{2}{*}{$\begin{array}{c}\text { BD 00001 } \\
(128 \times 128)\end{array}$} & $3 \times 3$ & 97.22 \\
\cline { 2 - 3 } & $7 \times 7$ & 91.66 \\
\hline BD 00002 & $11 \times 11$ & 94.72 \\
\cline { 2 - 3 }$(64 \times 64)$ & $3 \times 3$ & 99.30 \\
\cline { 2 - 3 } & $7 \times 7$ & 93.44 \\
\hline
\end{tabular}

The inclusion of the contrast and local orientation coherence distributions not only increased the overall classification accuracy but also we note that the discriminative power of the joint distribution (dominant orientation, contrast, orientation coherence) is significantly higher than the discriminative power of the dominant orientation distribution especially when applied to small texture images (see the classification results for database TC 00002). However, from the results depicted in Tables 2 and 3 we can draw some useful conclusions. The most important finding is that the distribution of the dominant orientations that are calculated at micro-level is appropriate to describe the texture at macro-level. In this regard, when the local orientation is calculated in a small neighbourhood this results in a fine sampling of the texture orientation. Conversely, when the local orientation is calculated for large texture units, the distribution of local orientations samples better the anisotropic character of the texture. The behaviour of this distribution of local orientations is interesting as it offers the possibility to sample the properties of the texture orientation at different sampling rates by calculating the joint distributions when the window sized is varied. Another important finding resulting from the experiments summarized in Tables 2 and 3 
is that the inclusion of the contrast and orientation coherence in a joint texture distribution improves the discriminative power offered by the distribution of the local orientations

Thus, the last experiment was conducted to evaluate whether the sampling of the orientation of the texture at different resolutions improves the overall classification results. In this regard, we have conducted a number of tests on Outex (Ojala et al, 2002-b) and Brodatz (Brodatz, 1966) databases where the texture orientation is sampled by the dominant orientation, contrast and orientation coherence distributions that are calculated for texture units with differing sizes in the range $3 \times 3$ to $11 \times 11$. The experimental results are depicted in Table 4

Table 4: Multi-resolution classification results. $(3 \times 3+7 \times 7$ joint distributions), $(3 \times 3+11 \times 11$ joint distributions $)$ and $(3 \times 3+7 \times 7+11 \times 11$ joint distributions $)$.

\begin{tabular}{|c|c|c|}
\hline Database & Window size & $\begin{array}{c}\text { Accuracy } \\
{[\%]}\end{array}$ \\
\hline \multirow{2}{*}{$\begin{array}{c}\text { TC } 00000 \\
(128 \times 128)\end{array}$} & $3 \times 3+7 \times 7$ & 97.91 \\
\cline { 2 - 3 } & $3 \times 3+11 \times 11$ & 96.66 \\
\hline \multirow{2}{*}{$\begin{array}{c}\text { TC } 00001 \\
(64 \times 64)\end{array}$} & $3 \times 7 \times 7+11 \times 11$ & 97.50 \\
\cline { 2 - 3 } & $3 \times 3+7 \times 7$ & 95.64 \\
\hline \multirow{2}{*}{$\begin{array}{c}\text { TC } 00002 \\
(32 \times 32)\end{array}$} & $3 \times 3 \times 3+7 \times 7$ & 92.80 \\
\cline { 2 - 3 } & $3 \times 3+7 \times 7+11 \times 11$ & 94.12 \\
\hline \multirow{2}{*}{$\begin{array}{c}\text { BD } 00000 \\
(256 \times 256)\end{array}$} & $3 \times 3+7 \times 7$ & 76.87 \\
\cline { 2 - 3 } & $3 \times 3+7 \times 7+11 \times 11$ & 69.06 \\
\hline \multirow{2}{*}{$\begin{array}{c}\text { BD } 00001 \\
(128 \times 128)\end{array}$} & $3 \times 3+7 \times 7$ & 100 \\
\cline { 2 - 3 } & $3 \times 3+11 \times 11$ & 100 \\
\cline { 2 - 3 } & $3 \times 3+7 \times 7+11 \times 11$ & 99.65 \\
\hline BD 00002 \\
$(64 \times 64)$ & $3 \times 3+7 \times 7$ & 98.26 \\
\cline { 2 - 3 } & $3 \times 3+11 \times 11$ & 94.62 \\
\cline { 2 - 3 } & $3 \times 3+7 \times 7+11 \times 11$ & 94.18 \\
\hline
\end{tabular}

The results shown in Table 4 clearly indicate that the classification accuracy increased when the texture orientation was sampled at different resolutions. From these results the best classification results have been achieved when the orientation of the texture is sampled by the $(3 \times 3+7 \times 7)$ joint distribution. We note that the classification results did not improve when the joint distribution was calculated for all resolutions $(3 \times 3,7 \times 7,11 \times 11)$. These experimental results indicate that in the classification process the local sampling of the texture orientation at microlevel has a greater discriminative power than the texture anisotropy that is sampled when the local orientation is estimated in larger neighbourhoods. The classification results are consistent when applied to two different texture databases and their discriminative power is comparable with that offered by other texture descriptors such as Local Binary Patterns (Ojala et al, 2002-a), Coordinated Cluster Representation (Sanchez et al, 2003) and the statistical features calculated from grey-level cooccurrence matrices (Haralick, 1979).

The computational complexity of the algorithm applied to calculate the dominant orientation, contrast and orientation coherence distributions from one texture image at different observation scales is depicted in Table 5. The experiments have been conducted using a $2.4 \mathrm{GHz}$ AMD X2 $4600 \mathrm{PC}$ and running Windows XP.

Table 5: Computational complexity of the algorithm applied to calculate the dominant orientation, orientation coherence and contrast distributions.

\begin{tabular}{|c|c|c|}
\hline Image Size & Window size & Time[sec] \\
\hline \multirow{3}{*}{$256 \times 256$} & $3 \times 3$ & 0.710 \\
\cline { 2 - 3 } & $7 \times 7$ & 0.920 \\
\cline { 2 - 3 } & $11 \times 11$ & 1.296 \\
\hline \multirow{3}{*}{$128 \times 128$} & $3 \times 3$ & 0.170 \\
\cline { 2 - 3 } & $7 \times 7$ & 0.219 \\
\cline { 2 - 3 } & $11 \times 11$ & 0.312 \\
\hline \multirow{3}{*}{$32 \times 32$} & $3 \times 3$ & 0.035 \\
\cline { 2 - 3 } & $7 \times 7$ & 0.046 \\
\cline { 2 - 3 } & $11 \times 11$ & 0.078 \\
\cline { 2 - 3 } & $3 \times 3$ & 0.016 \\
\hline & $7 \times 7$ & 0.031 \\
\hline
\end{tabular}

\section{CONCLUSIONS}

The aim of this paper was to evaluate the discriminative power of the local texture orientation in the classification process. The main contribution of this work resides in the methodology proposed to calculate the orientation of the texture at macro-level as the distribution of dominant orientations calculated for all texture units in the image that sample the texture orientation at micro-level. The distribution of the dominant local orientations in the 
image proved to be a robust texture feature when applied to classify large texture images, but its discriminative power was significantly lower when applied to the classification of small texture images. Thus, in this paper we proposed to complement the distribution of dominant orientations in the image with two additional distributions that measure the local contrast and local orientation coherence in the neighbourhood where the local dominant orientation was calculated. The inclusion of these two measures proved to be appropriate especially when the new joint descriptor was applied to the classification of texture databases containing images defined by small textures. Another important finding resulting from this investigation is the fact that the classification accuracy has improved when the orientation of the texture was sampled at different resolutions. One advantage of the texture extraction approach detailed in this paper over other texture descriptors such as Local Binary Patterns (Ojala et al, 2002-a) and grey-level co-occurrence matrices (Haralick, 1979) resides in the fact that the proposed orientation distributions can be further extended to be rotational invariant since they are $\pi$-periodic with respect to the orientation of the texture. The experimental results reported in this paper are promising and indicate that the distribution of local texture orientation is a robust feature that can describe the texture at macro-level. In our future studies we will further develop the proposed texture analysis technique to produce a rotation invariant representation and to analyse the effect of the noneven illumination on texture classification accuracy.

\section{ACKNOWLEDGEMENTS}

This work was funded in part by the Science Foundation Ireland (Research Frontiers Programme) and the OVPR-DCU Research Fellowship Programme. The authors would also like to thank Dr. Antonio Fernandez, University of Vigo, Spain, for his insightful comments on this work.

\section{REFERENCES}

Brodatz P., 1966. Textures: A Photographic Album for Artists and Designers. Dover Publications, New York, 1966.

Canny J., 1986. A computational approach to edge detection. IEEE Transactions on Pattern Analysis and Machine Intelligence, 8(6), pp. 679-698.
Chellappa R., Kashyap R.L., Manjunath B.S., 1998. Model based texture segmentation and classification, In The Handbook of Pattern Recognition and Computer Vision, C.H. Chen, L.F. Pau and P.S.P Wang (Editors) World Scientific Publishing.

Chang C., Lin C.J., 2001. LIBSVM: A library for support vector machines, www.csie.ntu.edu.tw/ cjlin/libsvm.

Dyer C.R., Hong T., Rosenfeld A., 1980. Texture classification using gray level co-occurrence based on edge maxima, IEEE Transactions on Systems, Man, and Cybernetics, 10, pp. 158-163.

Flores M.A., Leon L.A., 2003. Texture classification trough multiscale orientation histogram analysis, In Scale Space Methods in Computer Vision, LNCS 2695, pp. 479-493.

Germain C., Da Costa J.P., Lavialle O., Baylou P., 2003. Multiscale estimation of vector field anisotropy application to texture characterization, Signal Processing, 83, pp. 1487-1503.

Haralick R.M., 1979. Statistical and structural approaches to texture, In Proc of IEEE, 67, pp. 786-804.

Kass M, Witkin A, 1987. Analyzing oriented patterns, Computer Vision, Graphics, and Image Processing, 37(3), pp. 362-385.

Liu X., Wang D., 2003. Texture classification using spectral histograms", IEEE Transactions on Image Processing, 12(6), pp. 661-670.

Manjunath B.S., Ma W.Y., 1996. Texture features for browsing and retrieval of image data, IEEE Transactions on Pattern Analysis and Machine Intelligence, 18(8), pp. 837-842.

Marimon D., Touradj E., 2007. Orientation histogrambased matching for region tracking, In $8^{\text {th }}$ International Workshop on Image Analysis for Multimedia Interactive Services, Santorini, Greece.

Materka A., Strzelecki M., 1998. Texture analysis methods - A review, Technical Report, University of Lodz, Cost B11 Report.

Ojala T., Pietikainen M., Maenpaa T., 2002. Multiresolution gray-scale and rotation invariant texture classification with local binary patterns, IEEE Transactions on Pattern Analysis and Machine Intelligence, 24(7), pp. 971-987.

Ojala T., Maenpäa T., Pietikainen M., Viertola J., Kyllonen J., Huovinen S., 2002. Outex - a new framework for empirical evaluation of texture analysis algorithms, In Proc. 16th International Conference on Pattern Recognition, vol. 1, Quebec, Canada, pp. 701706.

Petrou M., Sevilla P.G., 2006. Image Processing: Dealing with Texture, John Wiley \& Sons, 2006.

Sanchez-Yanez R.E, Kurmyshev E.V., Fernandez A., 2003. One-class texture classifier in the CCR feature space, Pattern Recognition Letters, 24, pp. 1503-1511.

Zhou J., Xin L., Zhang D., 2003. Scale-orientation histogram for texture image retrieval, Pattern Recognition, 36, pp. 1061-1063. 